\title{
Pancreatite Auto-Imune: O Papel da Imagem no Diagnóstico
}

\author{
Auto-Immune Pancreatitis: The Diagnostic Role of Imaging Studies
}

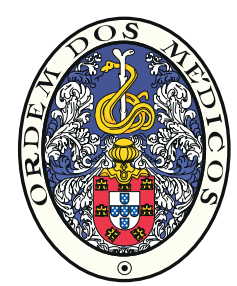

\author{
João RESSURREIÇÃO $₫ 1$, Maria BALI², Celso MATOS ${ }^{2}$ \\ Acta Med Port 2015 Sep-Oct;28(5):667-670
}

\section{RESUMO}

A pancreatite auto-imune é uma patologia incomum e subdiagnosticada. O seu quadro clínico é inespecífico, condicionando dificuldades diagnósticas. Os exames de imagem têm papel crucial no diagnóstico desta doença, assim como no controle da eficácia terapêutica. Com o objetivo de salientar a importância diagnóstica da imagiologia nesta patologia, os autores apresentam o caso de uma paciente com queixas abdominais inespecíficas não recentes, diagnosticado como pancreatite auto-imune após avaliação analítica e imagiológica. Com base neste diagnóstico foi instituída terapêutica com metilprednisolona oral, a qual foi bem-sucedida, com progressiva resolução clínica, laboratorial e imagiológica.

Palavras-chave: Doenças Auto-Imunes; Pancreatite; Ressonância Magnética; Tomografia Computorizada.

\section{ABSTRACT}

Auto-immune pancreatitis is an uncommon and underdiagnosed pathology. Its clinical picture is not specific and the diagnosis is therefore difficult. Imaging studies have a crucial role in the diagnostic process and also in the follow-up of treatment. With the objective of emphasizing the importance of imaging in the correct diagnosis of this pathology, the authors report the case of a patient with longterm unspecific abdominal complaints that was diagnosed as auto-immune pancreatitis after laboratorial and imaging investigation. Assuming this diagnosis it was instituted treatment with oral methylprednisolone, which succeeded, with progressive resolution of the clinical, laboratorial and imaging findings.

Keywords: Autoimmune Diseases; Magnetic Resonance Imaging; Pancreatitis; Tomography, X-Ray Computed.

\section{INTRODUÇÃO}

A pancreatite auto-imune (PAI) é uma patologia incomum que pode traduzir um processo primário do pâncreas ou, na sua forma mais comum, fazer parte de uma doença sistémica relacionada com IgG4. Por apresentar variantes patofisiológicas e na sua apresentação clínica e por poder mimetizar outras doenças com envolvimento pancreático, um elevado grau de suspeição é essencial para o seu diagnóstico e tratamento. ${ }^{1,2}$

\section{CASO CLÍNICO}

Doente do sexo masculino, caucasiano, 77 anos, com antecedentes clínicos de diabetes tipo II e hipertensão arterial, ambas mal-controladas apesar de tratamento apropriado. Sem outros antecedentes clínicos de relevo. Foi avaliado em consulta de Gastrenterologia por quadro de epigastralgias, anorexia, icterícia e perda ponderal de $5 \mathrm{~kg}$ com dois meses de evolução.

Neste contexto realizou estudo analítico que revelou padrão de colestase bastante marcado e aumento moderado da lipase (Tabela 1), tendo também realizado tomografia computorizada (TC) abdominal. A TC revelou um aspeto difusamente tumefato do pâncreas, com

Tabela 1 - Resultados analíticos

\begin{tabular}{lccc}
\hline & Dia 1 & Dia 15 & Valor de referência \\
\hline Bilirrubina total (mg/dL) & 4,8 & 0,56 & $<1,2$ \\
Fosfatase alcalina (U/L) & 830 & 138 & $56-119$ \\
Y-GT (U/L) & 1300 & 218 & 81 \\
Lipase (U/L) & 140 & 69 & $<70$ \\
IgG4 sérica (g/L) & 3,4 & 1,9 & 1,26 \\
\hline Y-GT: $\gamma$-glutamiltranspeptidase. & &
\end{tabular}

1. Serviço de Imagiologia. Centro Hospitalar de Vila Nova de Gaia/Espinho. Vila Nova de Gaia. Portugal.

2. Service de Radiologie. Hôpital Erasme. Université Libre de Bruxelles. Bruxelles. Belgique.

$\bowtie$ Autor correspondente: João Ressurreição. joaofres@gmail.com

Recebido: 15 de Outubro de 2014 - Aceite: 10 de Agosto de 2015 | Copyright @ Ordem dos Médicos 2015 


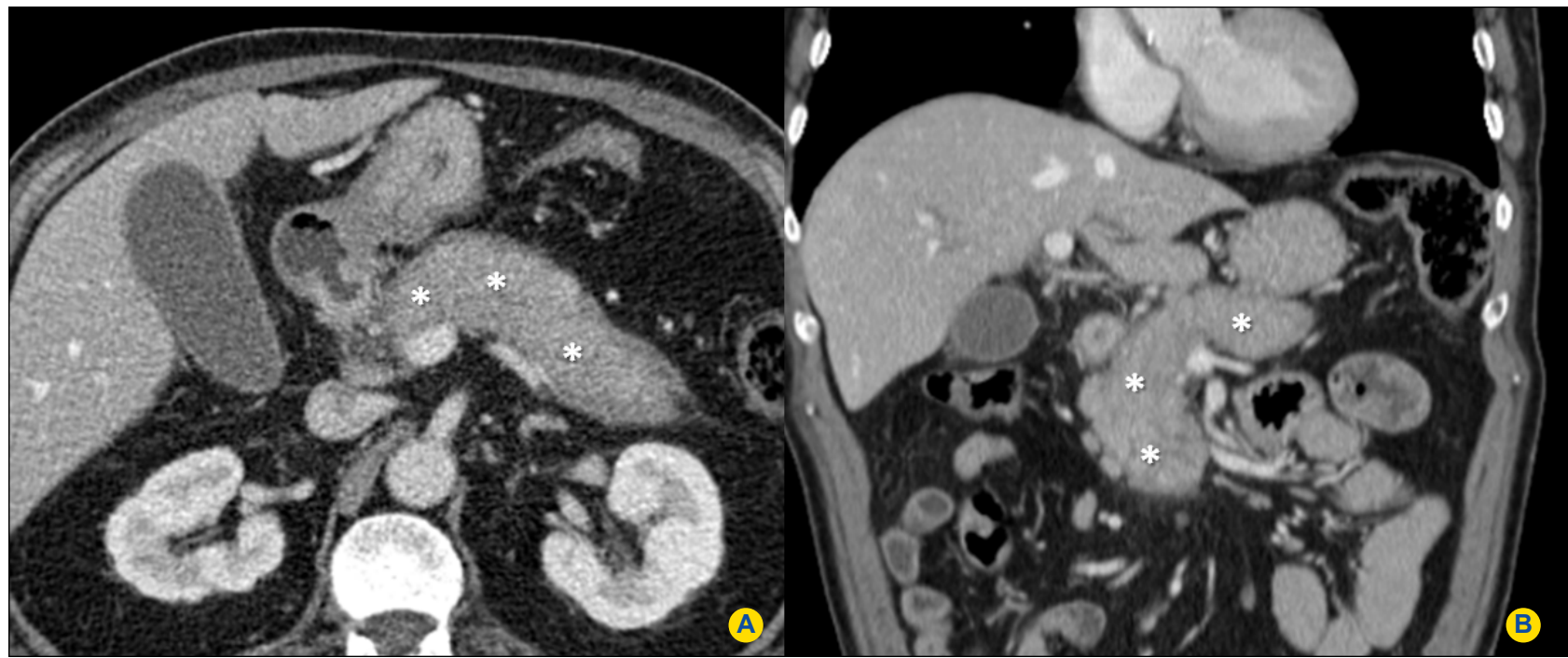

Figura 1 - TC com contraste. Pancreatite auto-imune, pré-tratamento. Imagem axial (A) e sagital (B) revelando pâncreas tumefacto $\left({ }^{*}\right)$, envolvido por halo hipodenso.

perda da habitual lobulação dos seus contornos e hiporrealce difuso. Constatou-se ainda discreto "halo" hipodenso periglandular e ausência de valorizável densificação da gordura peripancreática (Fig. 1). Foi também observada discreta proeminência das vias biliares. Perante estes achados, clínicos, laboratoriais e tomodensitométricos, foi levantada a hipótese diagnóstica de PAI, que foi corroborada pelo doseamento sérico de $\operatorname{lgG} 4$, com valores três vezes acima do normal (Tabela 1 ) e por achados de ressonância magnética abdominal com colangiopancreatografia associada (CPRM). Para além de confirmar os achados de TC, a CPRM demonstrou um canal pancreático principal fino e irregular e a existência de restrição do sinal de todo o pâncreas nas sequências de difusão (Fig. 2).

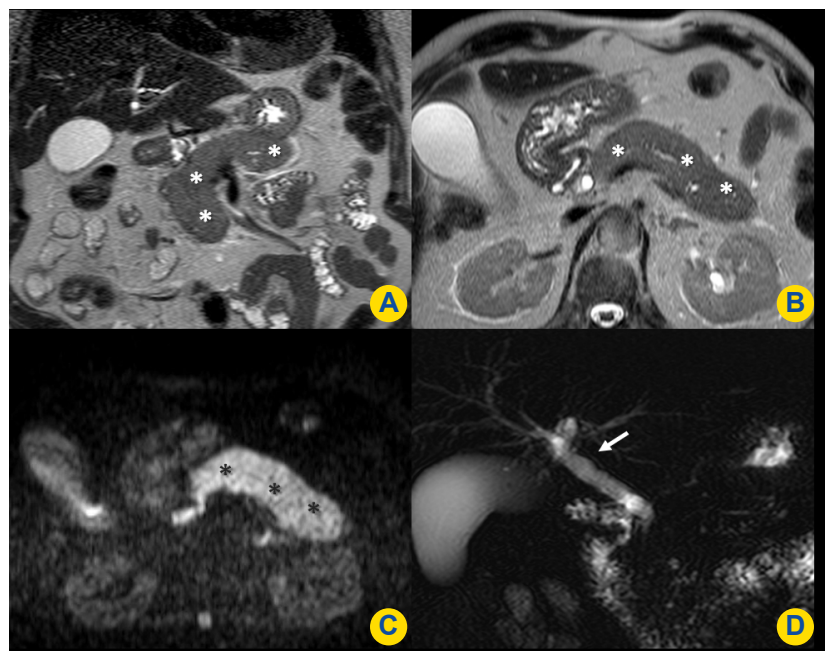

Figura 2 - RM. Pancreatite auto-imune, pré-tratamento. Sequências T2 nos planos coronal (A) e axial (B), difusão no plano axial $(b=1000)(C)$ e CPRM no plano coronal (D), revelando pâncreas tumefacto $(*)$, com irregularidade do canal pancreático principal e marcado hipersinal no estudo de difusão (C). Em (D) constata-se ligeira dilatação e irregularidade da via biliar principal (seta), que atinge um calibre de $10 \mathrm{~mm}$, a que se associa ligeira dilatação das vias biliares intra-hepáticas.
A investigação clínica não demonstrou envolvimento de outros sistemas orgânicos para além do sistema pancreático-biliar.

Efetuou tratamento com metilpredinosolona oral $(40 \mathrm{mg} /$ dia durante as primeiras 4 semanas), com avaliação clínica e laboratorial ao fim de duas semanas. Verificou-se melhoria clínica e laboratorial progressiva com atenuação significativa do padrão de colestase, normalização da lípase e redução importante dos valores de IgG4 (Tabela 1). Repetiu CPRM no final das quatro semanas, que revelou um pâncreas com normal morfologia parenquimatosa e ductal, ausência de restrição à difusão e normal calibre das vias biliares (Fig. 3). A resposta positiva ao tratamento permitiu efetuar uma redução progressiva da dose, prevendo-se

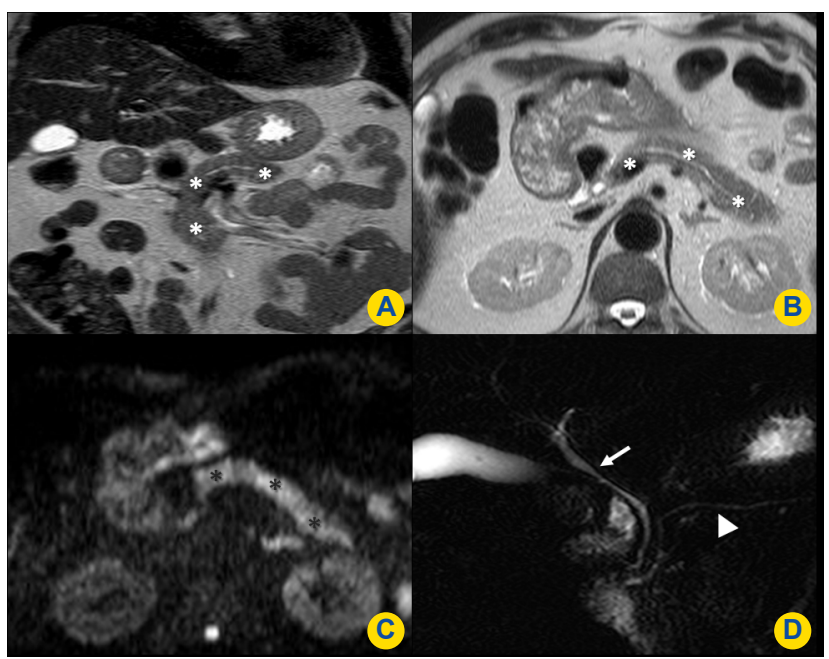

Figura 3 - RM. Pancreatite auto-imune, após 4 semanas de tratamento. Sequências T2 nos planos coronal $(A)$ e axial (B), difusão no plano axial $(b=1000)(C)$ e CPRM no plano coronal (D), revelando normalização das dimensões do pâncreas $\left(^{*}\right)$ e do seu sinal no estudo de difusão. Em (D) já não se observa dilatação das vias biliares (seta) e o canal pancreático principal apresenta normal calibre e é regular (cabeça de seta). 
suspensão completa ao fim de dois meses de tratamento. O doente prosseguirá vigilância clínica e laboratorial para avaliação de eventual recidiva.

\section{DISCUSSÃO}

A PAI é uma patologia fibroinflamatória crónica do pâncreas, de origem auto-imune. ${ }^{3,4,5}$ Embora se trate de uma doença incomum, de distribuição mundial, os valores de incidência e prevalência não são conhecidos com precisão pois trata-se de uma patologia geralmente subdiagnosticada. ${ }^{3,6-8}$ A sua etiopatogenia ainda não é completamente conhecida, mas é possível dividir a PAI em dois grupos: PAI do tipo 1 (PAl-1), o subtipo mais frequente, ${ }^{2}$ integrada num quadro de 'doença sistémica relacionada com IgG4' que, para além do pâncreas, pode envolver glândulas salivares, pulmões, vias biliares, rins, entre outros órgãos. Histologicamente o pâncreas apresenta um padrão inflamatório clássico do tipo esclerosante linfoplasmocitário, verificando-se acumulação de lgG4 no sangue, tecido pancreático e noutros órgãos; PAl do tipo 2 (PAI-2), patologia limitada ao pâncreas e que se caracteriza histologicamente por infiltração neutrofílica do epitélio ductal do pâncreas, o que leva à sua destruição. A PAl-2 não está associada a elevação dos níveis séricos / tecidulares de lgG4. 2,9

Clinicamente, a PAI cursa com icterícia obstrutiva, dor abdominal recorrente e emagrecimento, um quadro bastante inespecífico. A raridade da patologia, a falta de especificidade da clínica e o facto de existirem dois subtipos da doença faz com que o seu diagnóstico seja difícil e baseado num elevado grau de suspeição. Neste contexto, em 2011 foi criado um consenso multidisciplinar que reúne uma série de critérios para facilitar o diagnóstico desta doença: 1) imagiológicos (imagem do parênquima e canal pancreático); 2) serológicos (doseamento de lgG4); 3) histológicos (histologia pancreática); 4) avaliação da resposta à terapêutica com corticoides; 5) pesquisa de envolvimento extra-pancreático. ${ }^{10}$ Importa realçar que a histologia não é essencial para o diagnóstico de PAl-1, mas é essencial para o diagnóstico de PAI-2. ${ }^{10}$

O diagnóstico imagiológico baseia-se nos achados de TC e de RM. O quadro típico cursa com tumefação difusa do parênquima pancreático, com perda da lobulação dos seus contornos - 'pâncreas em salsicha' e envolvimento por um halo hipodenso em TC e hipointenso em todas as sequências de RM. O padrão de realce após administração de contraste endovenoso é variável, dependendo do grau de inflamação e fibrose do pâncreas, sendo que o aspeto típico é um realce progressivo que toma lugar numa fase mais tardia que o habitual. Outros achados característicos em RM são a marcada restrição do parênquima pancreático no estudo de difusão, assim como a redução de calibre e irregularidade do canal pancreático principal na CPRM. Com menor frequência pode ocorrer um envolvimento limitado da cabeça e/ou processo uncinado do pâncreas, com tumefação isolada destas regiões - forma focal da PAI, causando grande dificuldade no diagnóstico diferencial com adenocarcinoma pancreático..$^{1,11,12}$

O doseamento sérico de lgG4 é o marcador biológico mais utilizado para o diagnóstico de PAl-1, estimando-se que um valor superior a $1,4 \mathrm{~g} / \mathrm{L}$ tenho uma sensibilidade de $86 \%$ e uma especificidade de $91 \%$ para o diagnóstico de PAI no quadro clínico adequado. . $^{33,14}$

O tratamento da PAl está centrado na corticoterapia, estimando-se que dois terços dos pacientes respondam ao tratamento, embora cerca de $25 \%$ apresente recidiva e uma pequena proporção necessite de tratamento contínuo. $O$ regime terapêutico mais comum envolve tratamento com metilprednisolona durante 11 semanas: $40 \mathrm{mg} / \mathrm{dia}$ durante as primeiras 4 semanas com diminuição progressiva da dosagem durante as 7 semanas seguintes, a um ritmo de $5 \mathrm{mg} / \mathrm{semana}$. A resposta terapêutica pode começar a ocorrer ao fim de 2 semanas de tratamento. A ausência de resposta ao tratamento deve levar à consideração de outras hipóteses diagnósticas. ${ }^{6,14,15}$

\section{CONCLUSÃO}

A PAl é uma patologia incomum, embora subdiagnosticada. Os exames de imagem, os doseamentos laboratoriais (nomeadamente os valores séricos de lgG4) e a resposta à corticoterapia são a base do seu diagnóstico.

\section{CONFLITOS DE INTERESSE}

Os autores declaram não terem quaisquer conflitos de interesse.

\section{FONTE DE FINANCIAMENTO}

Os autores declaram que para a realização do presente trabalho não existiu qualquer financiamento.

\section{REFERÊNCIAS}

1. Bodily KD, Takahashi N, Fletcher JG, Fidler JL, Hough DM, Kawashima $A$, et al. Autoimmune pancreatitis: pancreatic and extrapancreatic imaging findings. AJR Am J Roentgenol. 2009;192:431-7.

2. Ketwaroo GA, Sheth S. Autoimmune pancreatitis. Gastroenterol Rep. 2013:1:27-32.

3. Khandelwal A, Shanbhogue AK, Takahashi N, Sandrasegaran K, Prasad SR. Recent advances in the diagnosis and management of autoimmune pancreatitis. AJR Am J Roentgenol. 2014;202:1007-21.

4. Kawaguchi K, Koike M, Tsuruta K, Okamoto A, Tabata I, Fujita N. Lymphoplasmacytic sclerosing pancreatitis with cholangitis: a variant of primary sclerosing cholangitis extensively involving pancreas. Hum Pathol. 1991;22:387-95.

5. Okazaki K, Kawa S, Kamisawa T, Naruse S, Tanaka S, Nishimori I, et al.
Clinical diagnostic criteria of autoimmune pancreatitis: revised proposal. J Gastroenterol. 2006;41:626-31.

6. Sah RP, Chari ST. Autoimmune pancreatitis: an update on classification, diagnosis, natural history and management. Curr Gastroenterol Rep. 2012;14:95-105.

7. Nishimori I, Tamakoshi A, Otsuki M, Research Committee on Intractable Diseases of the Pancreas, Ministry of Health, Labour, and Welfare of Japan. Prevalence of autoimmune pancreatitis in Japan from a nationwide survey in 2002. J Gastroenterol. 2007;42:6-8.

8. Sutton R. Autoimmune pancreatitis-also a Western disease. Gut. 2005;54:581-3.

9. Park $\mathrm{DH}$, Kim $\mathrm{MH}$, Chari ST. Recent advances in autoimmune pancreatitis. Gut. 2009;58:1680-9. 
10. Shimosegawa T, Chari ST, Frulloni L, Kamisawa T, Kawa S, MinoKenudson $M$, et al. International consensus diagnostic criteria for autoimmune pancreatitis: guidelines of the International Association of Pancreatology. Pancreas. 2011;40:352-8.

11. Irie $\mathrm{H}$, Honda $\mathrm{H}$, Baba $\mathrm{S}$, Kuroiwa $\mathrm{T}$, Yoshimitsu $\mathrm{K}$, Tajima $\mathrm{T}$, et al. Autoimmune pancreatitis: CT and MR characteristics. AJR Am J Roentgenol. 1998;170:1323-7.

12. Takahashi N, Fletcher JG, Fidler JL, Hough DM, Kawashima A, Chari ST. Dual-phase CT of autoimmune pancreatitis: a multireader study. AJR Am J Roentgenol. 2008;190:280-6.

13. Choi EK, Kim MH, Lee TY, Kwon S, Oh HC, Hwang CY, et al.
The sensitivity and specificity of serum immunoglobulin $G$ and immunoglobulin G4 levels in the diagnosis of autoimmune chronic pancreatitis: Korean experience. Pancreas. 2007;35:156-61.

14. Ghazale A, Chari ST, Smyrk TC, Levy MJ, Topazian MD, Takahashi $\mathrm{N}$. Value of serum IgG4 in the diagnosis of autoimmune pancreatitis and in distinguishing it from pancreatic cancer. Am J Gastroenterol. 2007;102:1646-53.

15. Moon SH, Kim MH, Park DH, Hwang CY, Park SJ, Lee SS, et al. Is a 2-week steroid trial after initial negative investigation for malignancy useful in differentiating autoimmune pancreatitis from pancreatic cancer? A prospective outcome study. Gut. 2008;57:1704.
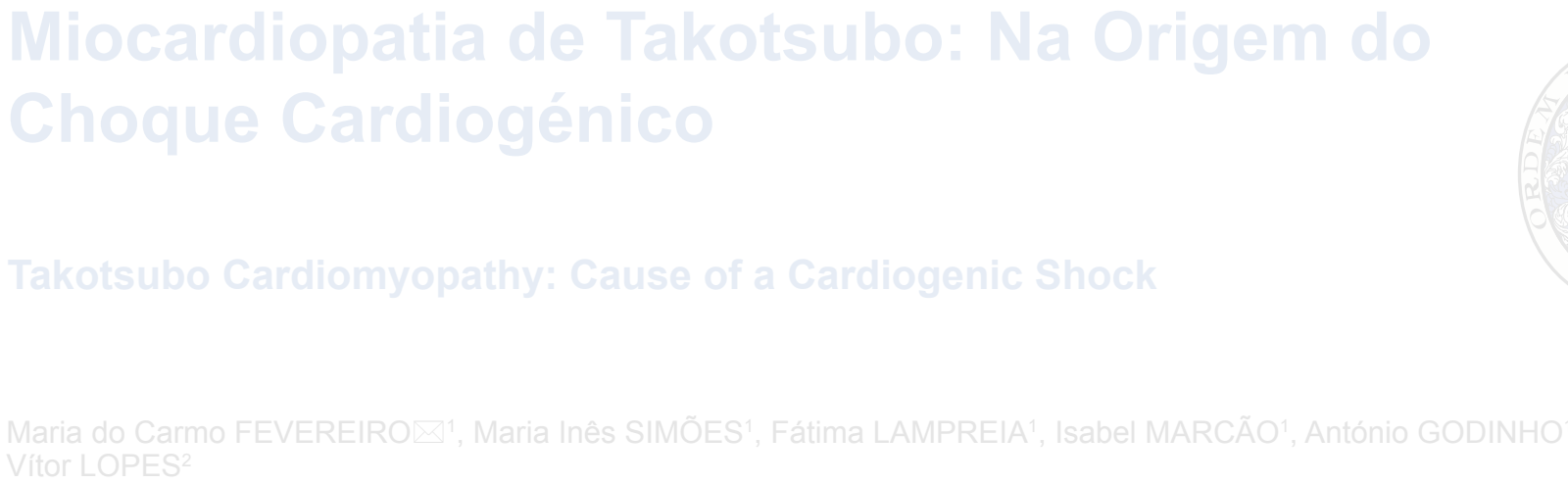

Acta Med Port 2015 Sep-Oct;28(5):670-673

\section{RESUIVO}
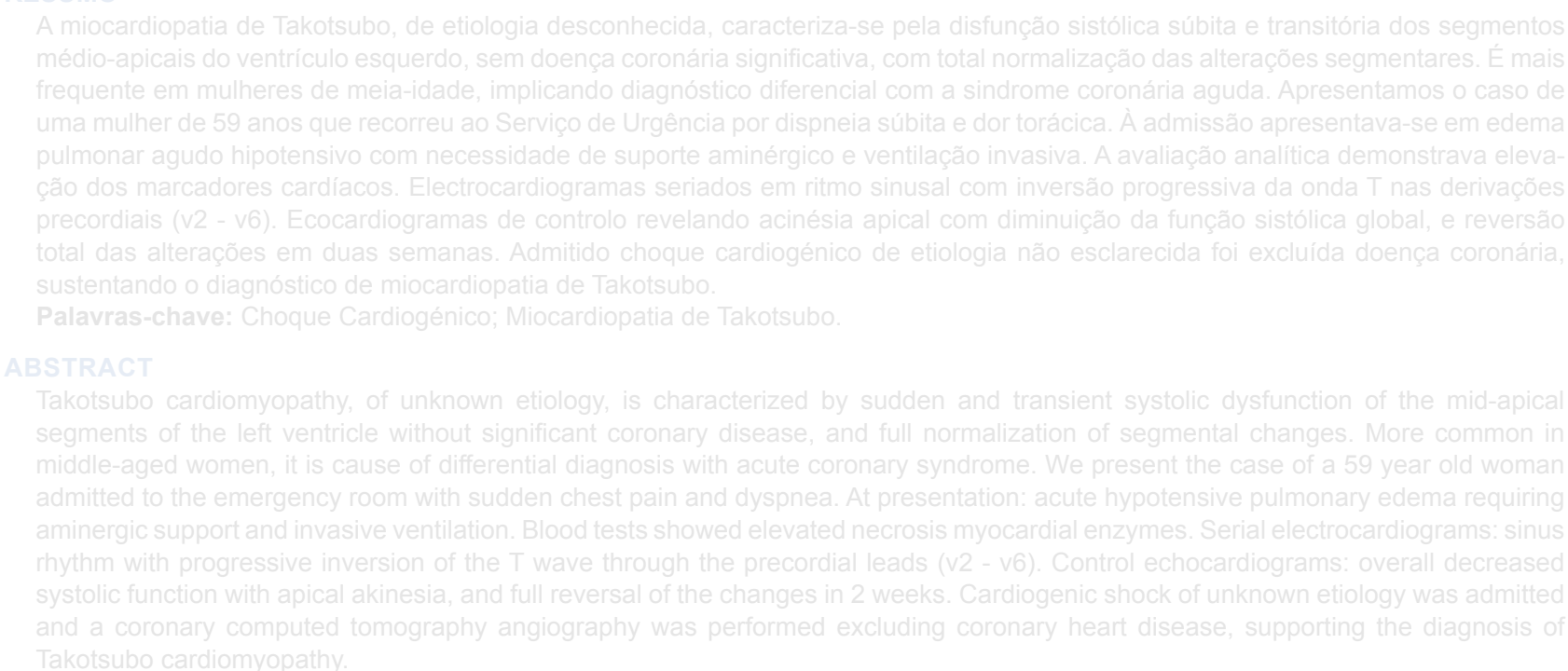

Keywords: Shock, Cardiogenic; Takotsubo Cardiomyopathy

INTRODUCÃ̃O 


\section{João RESSURREIÇÃO, Maria BALI, Celso MATOS \\ Pancreatite Auto-Imune: O Papel da Imagem no Diagnóstico}

Acta Med Port 2015:28:667-670

Publicado pela Acta Médica Portuguesa, a Revista Científica da Ordem dos Médicos

Av. Almirante Gago Coutinho, 151

1749-084 Lisboa, Portugal.

Tel: +351218428 215

E-mail: submissao@actamedicaportuguesa.com

www.actamedicaportuguesa.com

ISSN:0870-399X | e-ISSN: 1646-0758

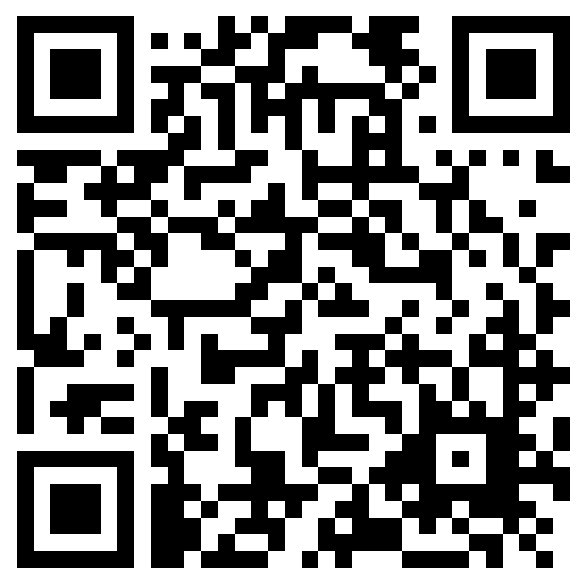

